Bangladesh J. Zool. 39(2): 187-194, 2011

\title{
EFFECT OF PLANTING DATE ON THE INCIDENCE OF EGGPLANT APHID, APHIS GOSSYPII GLOVER AND YIELD OF EGGPLANT
}

\author{
M. Matiur Rahman ${ }^{1}$, Pankoj Kumar Sarker² and Bidhan Chandra Das* \\ Department of Zoology, University of Rajshahi, Rajshahi-6205, Bangladesh
}

\begin{abstract}
Impact of planting dates on Aphis gossypii Glover infestation and yields of eggplants was studied in the field. The aphid population was very low (4.75-31.05 aphids/plant) on the first planted crops. Yield of first planting dates were higher (1.56-3.58 kg/plant), however; aphid population on the successive late planting crops gradually increased and reached to peaks at third planting dates (15.5196.46 aphids/plant). Yield of eggplant gradually decreased on successive late planting crops and lowest yield recorded for third planting crops (1.06-1.88 $\mathrm{kg} /$ plant).

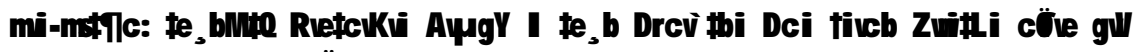

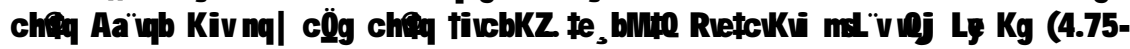

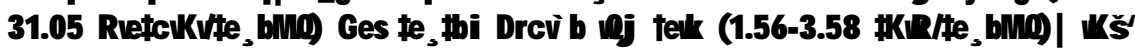

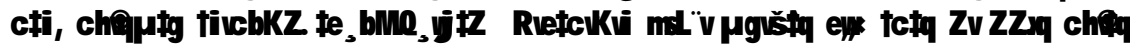

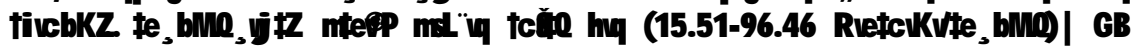

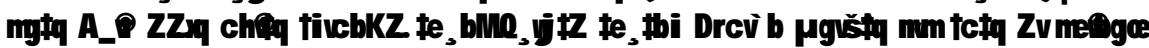
$n \neq Z+' \operatorname{vhva}(1.06-1.88 \neq K \mathbb{R} / \neq e, b M Q))$
\end{abstract}

Key words: Planting date, Aphis gossypii and eggplant.

\section{INTRODUCTION}

Eggplant (Solanum melongena L.) is an important solanaceous vegetable in many countries of Asia and Africa. It is a good source of minerals and vitamins in the tropical diets (Kumar et al. 2008). It is one of the most common, popular and principal vegetable crops grown in Bangladesh and other parts of the world. Among the different pests of this crop, the attack of the aphid, A. gossypii causes severe damage to eggplant (Gallo et al. 2002).

Seasonal control as well as date of planting has a good impact on yield and on pest incidence (Hossain et al. 2002). Early planting plants avoid aphid incidence (Singh 2006), plants are grown up before the pick time of aphid incidence and yields become higher than late planting (Ofuya 1997).

Crop maturation and growth rate of host plants are other important factors to escape an encounter of the aphid infestation and help to get maximum yield. Thus date of planting is an important tool of 'Cultural Control' as well as 'Integrated Pest Management System' (Gu et al. 2007).

${ }^{*}$ Corresponding author. E-mail: bcdzool@yahoo.com ${ }^{1}$ Department of Zoology, Narail Govt. Victoria Degree College, Narail, Bangladesh. ${ }^{2}$ Department of Zoology, Ghoraghat Degree College, Osmanpur, Dinajpur, Bangladesh. 
A number of workers conducted experiments on the impact of date of planting as well as cultural control of different crops in Bangladesh (Hossain et al. 2002) and abroad (Bhaduria et al. 1992, Gupta and Ram 1989, Reddy and Gowda 1987, Singh and Dhaliwal 2004). Information regarding the influence of A. gossypii infestation on the yield of eggplant in relation to different planting dates is not available in Bangladesh.

\section{OBJECTIVES}

The objects of the present study were: (i) to study the influence of planting date on the abundance of A. gossypii infesting the aforesaid varieties of eggplant crops, and (ii) to assess the yields of these varieties in relation to the aphid infestation.

\section{MATERIAL AND METHODS}

Experimental design: For the assessment of impact of planting dates on aphid infestation and yield on twelve varieties of eggplant for three planting dates of three consecutive seasons (2005-2006, 2006-2007 and 2007-2008), the same experimental fields were used in the research field of Zoology Department, Rajshahi University campus. Altogether 36 blocks (each 1.35 sq. meters) each contained three replicated plots were used. Twelve varieties of eggplants, viz. Kajla, Uttara, Nayantara (BARI released varieties), Singnath, Islampuri, Jhumka, Chakri, Khotkhotia, Ananda, Ullash and Tabla (local varieties) were selected and were planted at different dates- $12^{\text {th }}$ September, $2^{\text {nd }}$ October and 22nd October. All plots received equal amount of fertilizers and two times of irrigations and more or less uniform mulching.

Data collection: Data were collected on the number of aphids per plant on twelve varieties of eggplants for three planting dates and three seasons, once in a week. From each plant, three leaves (one each from top, middle and bottom) were selected for the aphid counting. The alate, nymphs and apterae were counted at weekly intervals for sixteen weeks.

Yield assessment: The eggplant fruits from each plant (in $\mathrm{kg}$ ) were collected every 5-7 days interval. The harvesting was continued till the end of the fruiting. Only mature and marketable size fruits were harvested and the harvested fruits were weighted to assess yields of each varieties and planting dates. Statistical analyses were performed to asses the differences in the yields in different planting dates and varieties.

Data analysis: Analyses of variance (ANOVA) were done to find out difference among the yields of twelve varieties of eggplant in respect of planting dates. The yields were compared using Duncan's Multiple Range Test (DMRT). Correlations 
of coefficients ('r' values) were determined to examine the type of relationships between the aphid populations and the yields.

\section{RESULTS AND DISCUSSION}

Mean populations of $A$. gossypii and yields of three planting dates of twelve varieties of eggplant for three consecutive seasons are presented in Tables 1 and 2. The coefficient of correlations (' $r$ ' values) and regression equations ( $y=a+b x)$ of aphid population and yield of twelve varieties of eggplants for three planting dates of three consecutive seasons are provided in Table 3.

In all three seasons, aphid population was very low (Table 1) on the eggplants of first planting dates and produced maximum yields for all the varieties (Table 2). The aphid populations on the eggplants of second and third planting dates gradually increased and their yields were also gradually decreased. Significant difference $(\mathrm{P}<0.05, \mathrm{P}<0.01$ and $\mathrm{p}<0.001)$ existed among the yields of three planting dates for twelve varieties of eggplant (Table 3 ) for all the seasons.

Highest yields $3.58,2.84$ and $2.97 \mathrm{~kg} /$ plant obtained on the eggplant variety, Kazla and lowest yields $1.28,1.07$ and $1.06 \mathrm{~kg} /$ plant found in the variety Islampuri were recorded for the seasons 2005-6, 2006-7 and 2007-8, respectively (Table 2).

In season 2005-2006, significant relationships $(\mathrm{p}<0.05, \mathrm{p}<0.01)$ were found between aphid populations and the yields of the varieties Kazla, Chakri, Khotkhotia, Ananda and Ullash; however, for the seasons 2006-2007 and 20072008 relationships between the aphid population and the yields of all twelve varieties of eggplants were significant $(p<0.01, p<0.001)$ (Table 3 ).

From the above results it is clear that early planting eggplants produced highest yield for all the varieties. During this time aphid infestation was very low. The eggplants of first planting date escaped the peak period of aphid infestation which occurred gradually during the third planting dates and produced the lowest amount of fruits. Planting date had significant influence on the activities of A. gossypii.

A number of workers reported the range of yields for eggplant crop. They got the yield 10.4-16.8 ton/ha (Soe 1999), 9.9-16.8 ton/ha (Wuzhong 2002) and 76.5291.67 ton/ha (Siddiky et al. 2007). But, they did not mention whether their crops were infested by aphids or not. They also did not mention their planting date. The effect of different dates of planting on the yield of three eggplant cultivers, Pusa Kranti, pusa Purple and Malapur Thornless was studied by Reddy and Gowda (1987) and they reported that planting in August gave the highest yields. 
Table 1. Mean aphid population (per plant) of twelve varieties of eggplant at three planting dates for three seasons 2005-2006, 2006-2007 and 2007-2008.

\begin{tabular}{|c|c|c|c|c|}
\hline \multirow{2}{*}{\multicolumn{2}{|c|}{ Eggplant varieties }} & \multicolumn{3}{|c|}{ Mean aphid population (per plant) } \\
\hline & & \multirow{2}{*}{$\begin{array}{c}\begin{array}{c}\text { First Planting } \\
\text { (Mean } \pm \mathrm{SE} \text { ) }\end{array} \\
4.75 \pm 1.04\end{array}$} & \multirow{2}{*}{$\begin{array}{c}\begin{array}{c}\text { Second Planting } \\
(\text { Mean } \pm \mathrm{SE})\end{array} \\
14.42 \pm 2.88\end{array}$} & \multirow{2}{*}{$\begin{array}{c}\begin{array}{c}\text { Third Planting } \\
(\text { Mean } \pm \mathrm{SE})\end{array} \\
17.14 \pm 3.23\end{array}$} \\
\hline $2005-06$ & Kazla & & & \\
\hline & Uttara & $7.03 \pm 1.54$ & $25.96 \pm 5.61$ & $21.98 \pm 4.18$ \\
\hline & Nayantara & $7.85 \pm 1.52$ & $28.66 \pm 5.90$ & $23.82 \pm 4.73$ \\
\hline & Singnath & $16.87 \pm 3.59$ & $50.14 \pm 9.96$ & $42.12 \pm 9.10$ \\
\hline & Islampuri & $27.24 \pm 5.12$ & $84.69 \pm 16.29$ & $67.14 \pm 13.94$ \\
\hline & Jhumka & $23.49 \pm 5.11$ & $78.34 \pm 15.45$ & $55.30 \pm 12.70$ \\
\hline & Chakri & $13.17 \pm 3.18$ & $38.27 \pm 8.43$ & $38.15 \pm 8.06$ \\
\hline & Muktakeshi & $24.72 \pm 4.76$ & $78.19 \pm 15.25$ & $60.59 \pm 13.30$ \\
\hline & Khotkhotia & $19.91 \pm 4.28$ & $65.78 \pm 12.20$ & $56.71 \pm 12.59$ \\
\hline & Ananda & $13.62 \pm 3.61$ & $34.48 \pm 8.05$ & $34.37 \pm 8.52$ \\
\hline & Ullash & $8.77 \pm 1.86$ & $28.37 \pm 5.94$ & $28.27 \pm 6.37$ \\
\hline & Tabla & $23.10 \pm 4.43$ & $77.19 \pm 15.52$ & $56.49 \pm 12.72$ \\
\hline \multicolumn{2}{|r|}{ Mean } & $15.88 \pm 1.942$ & $50.37 \pm 6.276$ & $41.84 \pm 4.258$ \\
\hline \multirow{13}{*}{$\begin{array}{l}2006-07 \\
\text { season }\end{array}$} & Kazla & $6.10 \pm 1.13$ & $14.87 \pm 2.45$ & $15.51 \pm 2.39$ \\
\hline & Uttara & $7.69 \pm 1.33$ & $22.23 \pm 3.77$ & $22.87 \pm 3.71$ \\
\hline & Nayantara & $9.74 \pm 1.59$ & $24.10 \pm 3.84$ & $25.03 \pm 3.94$ \\
\hline & Singnath & $16.39 \pm 2.68$ & $39.52 \pm 7.21$ & $44.02 \pm 7.15$ \\
\hline & Islampuri & $31.05 \pm 4.18$ & $62.26 \pm 12.30$ & $70.47 \pm 13.74$ \\
\hline & Jhumka & $21.46 \pm 3.64$ & $52.46 \pm 10.24$ & $57.10 \pm 11.32$ \\
\hline & Chakri & $14.56 \pm 2.51$ & $41.20 \pm 6.68$ & $43.20 \pm 7.41$ \\
\hline & Muktakeshi & $27.48 \pm 4.11$ & $56.94 \pm 11.91$ & $63.05 \pm 13.60$ \\
\hline & Khotkhotia & $23.34 \pm 3.74$ & $53.44 \pm 11.11$ & $58.05 \pm 11.84$ \\
\hline & Ananda & $19.75 \pm 3.03$ & $47.33 \pm 7.96$ & $51.81 \pm 9.53$ \\
\hline & Ullash & $11.73 \pm 1.82$ & $27.01 \pm 4.76$ & $32.38 \pm 5.68$ \\
\hline & Tabla & $24.21 \pm 3.69$ & $62.60 \pm 11.46$ & $56.79 \pm 11.30$ \\
\hline & Mean & $17.79 \pm 2.011$ & $42.00 \pm 4.132$ & $45.02 \pm 4.3$ \\
\hline \multirow{13}{*}{$\begin{array}{l}2007-08 \\
\text { season }\end{array}$} & Kazla & $5.14 \pm 1.03$ & $18.77 \pm 2.48$ & $20.67 \pm 2.48$ \\
\hline & Uttara & $6.84 \pm 1.45$ & $27.99 \pm 3.97$ & $27.94 \pm 3.97$ \\
\hline & Nayantara & $8.32 \pm 1.44$ & $30.64 \pm 4.19$ & $30.26 \pm 4.14$ \\
\hline & Singnath & $12.39 \pm 1.82$ & $48.90 \pm 7.69$ & $54.16 \pm 7.47$ \\
\hline & Islampuri & $21.34 \pm 2.69$ & $79.08 \pm 12.52$ & $96.46 \pm 14.14$ \\
\hline & Jhumka & $16.66 \pm 2.38$ & $67.71 \pm 11.12$ & $78.43 \pm 12.32$ \\
\hline & Chakri & $11.26 \pm 2.15$ & $51.35 \pm 6.52$ & $55.97 \pm 7.77$ \\
\hline & Muktakeshi & $18.53 \pm 2.61$ & $72.93 \pm 12.81$ & $88.64 \pm 14.48$ \\
\hline & Khotkhotia & $16.01 \pm 2.41$ & $60.05 \pm 11.34$ & $78.89 \pm 12.91$ \\
\hline & Ananda & $13.37 \pm 2.24$ & $58.40 \pm 8.56$ & $64.46 \pm 9.82$ \\
\hline & Ullash & $9.32 \pm 1.77$ & $32.16 \pm 4.27$ & $43.11 \pm 6.32$ \\
\hline & Tabla & $17.89 \pm 2.57$ & $65.26 \pm 13.10$ & $79.65 \pm 11.59$ \\
\hline & Mean & $13.29 \pm 1.272$ & $51.10 \pm 4.903$ & $59.84 \pm 6.319$ \\
\hline
\end{tabular}


Table 2. Mean yields (kg/plant) of twelve varieties of eggplant at three planting dates for three seasons: 2005-2006, 2006-2007 and 2007-2008.

\begin{tabular}{|c|c|c|c|c|c|}
\hline \multirow{2}{*}{\multicolumn{2}{|c|}{ Eggplant varieties }} & \multicolumn{4}{|c|}{ Mean yields (kg/plant) } \\
\hline & & \multirow{2}{*}{$\begin{array}{c}\begin{array}{c}\text { First Planting } \\
(\text { Mean } \pm \mathrm{SE})\end{array} \\
3.58 \pm 0.028 \mathrm{Aa}\end{array}$} & \multirow{2}{*}{ 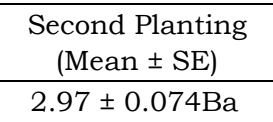 } & \multirow{2}{*}{$\begin{array}{c}\text { Third Planting } \\
(\text { Mean } \pm \mathrm{SE})\end{array}$} & \multirow{2}{*}{$\frac{\mathrm{p} \text {-values }}{0.000}$} \\
\hline Season: & Kazla & & & & \\
\hline 2005-06 & Uttara & $3.01 \pm 0.323 \mathrm{Ab}$ & $2.82 \pm 0.037 \mathrm{Ab}$ & $1.77 \pm 0.061 \mathrm{Ba}$ & 0.000 \\
\hline & Nayantara & $2.73 \pm 0.048 \mathrm{Ab}$ & $2.61 \pm 0.052 \mathrm{Ab}$ & $1.68 \pm 0.041 \mathrm{Bb}$ & 0.000 \\
\hline & Singnath & $2.68 \pm 0.02 \mathrm{Ab}$ & $2.39 \pm 0.041 \mathrm{Bc}$ & $1.42 \pm 0.031 \mathrm{Cde}$ & 0.000 \\
\hline & Islampuri & $1.79 \pm 0.022 \mathrm{Af}$ & $1.63 \pm 0.038 \mathrm{Bef}$ & $1.28 \pm 0.041 \mathrm{Bcd}$ & 0.006 \\
\hline & Jhumka & $1.95 \pm 0.058 \mathrm{Ae}$ & $1.76 \pm 0.042 \mathrm{ABdef}$ & $1.32 \pm 0.068 \mathrm{Bbc}$ & 0.037 \\
\hline & Chakri & $2.71 \pm 0.027 \mathrm{Ab}$ & $2.52 \pm 0.05 \mathrm{Bc}$ & $1.58 \pm 0.031 \mathrm{Ccd}$ & 0.000 \\
\hline & Muktakeshi & $1.78 \pm 0.018 \mathrm{Af}$ & $1.62 \pm 0.034 \mathrm{Bf}$ & $1.41 \pm 0.029 \mathrm{Ccde}$ & 0.001 \\
\hline & Khotkhotia & $1.98 \pm 0.045$ Ade & $1.82 \pm 0.026 \mathrm{Bde}$ & $1.51 \pm 0.035 \mathrm{Cc}$ & 0.000 \\
\hline & Ananda & $2.23 \pm 0.042 \mathrm{Acd}$ & $1.97 \pm 0.038 \mathrm{Bd}$ & $1.53 \pm 0.064 \mathrm{Ccde}$ & 0.000 \\
\hline & Ullash & $2.29 \pm 0.029 \mathrm{Ac}$ & $1.92 \pm 0.054 \mathrm{Bde}$ & $1.56 \pm 0.063 \mathrm{Bbc}$ & 0.001 \\
\hline & Tabla & $1.87 \pm 0.021 \mathrm{Af}$ & $1.72 \pm 0.025$ Adef & $1.45 \pm 0.06 \mathrm{Be}$ & 0.001 \\
\hline & 'P' values & 0.0 & 0.0 & 0.0 & \\
\hline & Mean & $2.38 \pm 0.141$ & $2.14 \pm 0.121$ & $1.53 \pm 0.044$ & \\
\hline \multirow{14}{*}{$\begin{array}{l}\text { Season: } \\
2006-07\end{array}$} & Kazla & $2.84 \pm 0.03 \mathrm{Aa}$ & $1.95 \pm 0.041 \mathrm{Ba}$ & $1.68 \pm 0.042 \mathrm{Ca}$ & 0.000 \\
\hline & Uttara & $2.73 \pm 0.026 \mathrm{Aa}$ & $1.76 \pm 0.043 \mathrm{Bab}$ & $1.66 \pm 0.038 \mathrm{Ba}$ & 0.000 \\
\hline & Nayantara & $2.58 \pm 0.04 \mathrm{Ab}$ & $1.68 \pm 0.036 \mathrm{Bbc}$ & $1.37 \pm 0.026 \mathrm{Cb}$ & 0.000 \\
\hline & Singnath & $2.28 \pm 0.057 \mathrm{Ac}$ & $1.41 \pm 0.033 \mathrm{Bcd}$ & $1.29 \pm 0.037 \mathrm{Cbc}$ & 0.000 \\
\hline & Islampuri & $1.56 \pm 0.031 \mathrm{Ae}$ & $1.31 \pm 0.032 \mathrm{Bd}$ & $1.07 \pm 0.026 \mathrm{Ccd}$ & 0.002 \\
\hline & Jhumka & $1.71 \pm 0.041$ Ade & $1.37 \pm 0.045 \mathrm{Bd}$ & $1.11 \pm 0.037 \mathrm{Ccd}$ & 0.000 \\
\hline & Chakri & $2.46 \pm 0.056 \mathrm{Ab}$ & $1.54 \pm 0.036 \mathrm{Bbcd}$ & $1.33 \pm 0.036 \mathrm{Bb}$ & 0.001 \\
\hline & Muktakeshi & $1.59 \pm 0.023 \mathrm{Ae}$ & $1.38 \pm 0.037$ Acd & $1.15 \pm 0.044 \mathrm{Bd}$ & 0.002 \\
\hline & Khotkhotia & $1.77 \pm 0.031 \mathrm{Ad}$ & $1.47 \pm 0.036 \mathrm{Bcd}$ & $1.23 \pm 0.04 \mathrm{Bbc}$ & 0.002 \\
\hline & Ananda & $1.92 \pm 0.033 \mathrm{Ad}$ & $1.51 \pm 0.068 \mathrm{Bcd}$ & $1.36 \pm 0.039 \mathrm{Bb}$ & 0.002 \\
\hline & Ullash & $1.86 \pm 0.042 \mathrm{Ad}$ & $1.53 \pm 0.056 \mathrm{ABbcd}$ & $1.37 \pm 0.049 \mathrm{Bb}$ & 0.024 \\
\hline & Tabla & $1.74 \pm 0.022$ Ade & $1.43 \pm 0.04 \mathrm{Bd}$ & $1.33 \pm 0.048 \mathrm{Bbcd}$ & 0.001 \\
\hline & 'P' values & 0.0 & 0.0 & 0.0 & \\
\hline & Mean & $2.22 \pm 0.125$ & $1.52 \pm 0.046$ & $1.32 \pm 0.047$ & \\
\hline \multirow{14}{*}{$\begin{array}{l}\text { Season: } \\
\text { 2007-08 }\end{array}$} & Kazla & $2.97 \pm 0.061 \mathrm{Aa}$ & $1.91 \pm 0.033 \mathrm{Ba}$ & $1.63 \pm 0.037 \mathrm{Ca}$ & 0.000 \\
\hline & Uttara & $2.82 \pm 0.026 \mathrm{Aab}$ & $1.7 \pm 0.034 \mathrm{Bb}$ & $1.62 \pm 0.023 \mathrm{Ba}$ & 0.000 \\
\hline & Nayantara & $2.61 \pm 0.045 \mathrm{Ab}$ & $1.62 \pm 0.026 \mathrm{Bb}$ & $1.36 \pm 0.024 \mathrm{Cb}$ & 0.000 \\
\hline & Singnath & $2.39 \pm 0.045 \mathrm{Ac}$ & $1.35 \pm 0.027 \mathrm{Bbcd}$ & $1.26 \pm 0.026 \mathrm{Bbc}$ & 0.000 \\
\hline & Islampuri & $1.64 \pm 0.032 \mathrm{Af}$ & $1.26 \pm 0.036 \mathrm{Bcde}$ & $1.06 \pm 0.02 \mathrm{Cd}$ & 0.005 \\
\hline & Jhumka & $1.78 \pm 0.031 \mathrm{Ade}$ & $1.26 \pm 0.051 \mathrm{Be}$ & $1.09 \pm 0.032 \mathrm{Bcd}$ & 0.000 \\
\hline & Chakri & $2.53 \pm 0.045 \mathrm{Ab}$ & $1.46 \pm 0.037 \mathrm{Bbcd}$ & $1.31 \pm 0.035 \mathrm{Bb}$ & 0.000 \\
\hline & Muktakeshi & $1.64 \pm 0.034 \mathrm{Af}$ & $1.33 \pm 0.042 \mathrm{Bde}$ & $1.12 \pm 0.032 \mathrm{Bd}$ & 0.005 \\
\hline & Khotkhotia & $2.18 \pm 0.027$ Ade & $1.4 \pm 0.038 \mathrm{Bbcd}$ & $1.12 \pm 0.041 \mathrm{Ccd}$ & 0.000 \\
\hline & Ananda & $1.97 \pm 0.041 \mathrm{Acd}$ & $1.45 \pm 0.057 \mathrm{Bbcd}$ & $1.33 \pm 0.028 \mathrm{Bb}$ & 0.003 \\
\hline & Ullash & $1.91 \pm 0.052 \mathrm{Acd}$ & $1.47 \pm 0.043 \mathrm{Bbc}$ & $1.33 \pm 0.037 \mathrm{Bb}$ & 0.016 \\
\hline & Tabla & $1.72 \pm 0.018 \mathrm{Aef}$ & $1.36 \pm 0.027 \mathrm{Bcde}$ & $1.29 \pm 0.044 \mathrm{Bbc}$ & 0.000 \\
\hline & 'P' values & 0.0 & 0.0 & 0.0 & \\
\hline & Mean & $2.18 \pm 0.118$ & $1.46 \pm 0.048$ & $1.29 \pm 0.046$ & \\
\hline
\end{tabular}

*Means followed by the same letter did not differ significantly at the $\mathrm{P}<0.05, \mathrm{P}<0.01$ and $\mathrm{P}<0.001$ by DMRT. Small and capital letter indicate column and row respectively. 
Table 3. Correlation coefficients (' $r$ ' values) and regression lines between aphid population (AP) and yield of twelve varieties of eggplant crops of three planting dates for the seasons 2005-2006, 2006-2007 and 2007-2008.

\begin{tabular}{|c|c|c|c|}
\hline \multirow{13}{*}{$\begin{array}{l}2005-06 \\
\text { season }\end{array}$} & Variables $(\mathrm{N}=9)$ & $\begin{array}{l}\text { Coefficient of } \\
\text { correlation (r) }\end{array}$ & $\begin{array}{l}\text { Regression equation } \\
(y=a+b x)\end{array}$ \\
\hline & AP \& yield (Kazla) & $\mathrm{r}=-0.87161^{*}$ & $y=4.2071-0.1212 x$ \\
\hline & AP \& yield (Uttara) & $r=-0.33775$ & $y=2.8408-0.0213 x$ \\
\hline & AP \& yield (Nayantara) & $r=-0.32777$ & $y=2.7105-0.0179 x$ \\
\hline & AP \& yield (Singnath) & $r=-0.51928$ & $y=2.8597-0.0194 x$ \\
\hline & AP \& yield (Islampuri) & $r=-0.38987$ & $y=1.7899-0.0037 x$ \\
\hline & AP \& yield (Jhumka) & $r=-0.60017$ & $y=2.1855-0.0081 x$ \\
\hline & AP \& yield (Chakri) & $r=-0.70785^{*}$ & $y=3.1026-0.0293 x$ \\
\hline & AP \& yield (Muktakeshi) & $r=-0.50289$ & $y=1.8069-0.004 x$ \\
\hline & AP \& yield (Khotkhotia) & $r=-0.72136^{*}$ & $y=2.2187-0.0094 x$ \\
\hline & AP \& yield (Ananda) & $r=-0.82655^{* *}$ & $y=2.6813-0.0287 x$ \\
\hline & AP \& yield (Ullash) & $r=-0.73982^{*}$ & $y=2.566-0.0299 x$ \\
\hline & AP \& yield (Tabla) & $r=-0.41257$ & $y=1.8843-0.0038 x$ \\
\hline \multirow{12}{*}{$\begin{array}{l}2006-07 \\
\text { season }\end{array}$} & AP \& yield (Kazla) & $\mathrm{r}=-0.98513^{* * *}$ & $y=3.4347-0.1029 x$ \\
\hline & AP \& yield (Uttara) & $r=-0.98845^{* * *}$ & $y=3.325-0.0717 x$ \\
\hline & AP \& yield (Nayantara) & $r=-0.98533^{* * *}$ & $y=3.4415-0.0774 x$ \\
\hline & AP \& yield (Singnath) & $r=-0.96698^{* * *}$ & $y=2.785-0.0346 x$ \\
\hline & AP \& yield (Islampuri) & $\mathrm{r}=-0.92158^{* * *}$ & $y=1.9103-0.0112 x$ \\
\hline & AP \& yield (Jhumka) & $r=-0.81835^{* *}$ & $y=1.998-0.0132 x$ \\
\hline & AP \& yield (Chakri) & $r=-0.97076^{* * *}$ & $y=2.7274-0.031 x$ \\
\hline & AP \& yield (Muktakeshi) & $r=-0.88596^{* *}$ & $y=1.9413-0.0122 x$ \\
\hline & AP \& yield (Khotkhotia) & $r=-0.92225^{* * *}$ & $y=2.0443-0.0123 x$ \\
\hline & AP \& yield (Ananda) & $r=-0.96387^{* * *}$ & $y=2.2867-0.0193 x$ \\
\hline & AP \& yield (Ullash) & $r=-0.83492^{* *}$ & $y=2.1997-0.026 x$ \\
\hline & AP \& yield (Tabla) & $\mathrm{r}=-0.84398^{* *}$ & $y=1.9889-0.0111 x$ \\
\hline \multirow{12}{*}{$\begin{array}{l}2007-08 \\
\text { season }\end{array}$} & AP \& yield (Kazla) & $r=-0.97741^{* * *}$ & $y=3.4684-0.0834 x$ \\
\hline & AP \& yield (Uttara) & $r=-0.99774 * * *$ & $y=3.235-0.0582 x$ \\
\hline & AP \& yield (Nayantara) & $r=-0.97097^{* * *}$ & $y=3.1132-0.0521 x$ \\
\hline & AP \& yield (Singnath) & $r=-0.99039 * * *$ & $y=2.7918-0.0292 x$ \\
\hline & AP \& yield (Islampuri) & $r=-0.94422^{* * *}$ & $y=1.7072-0.0064 x$ \\
\hline & AP \& yield (Jhumka) & $r=-0.89999 * * *$ & $y=1.9347-0.0096 x$ \\
\hline & AP \& yield (Chakri) & $r=-0.98262^{* * *}$ & $y=2.9912-0.0299 x$ \\
\hline & AP \& yield (Muktakeshi) & $r=-0.88135^{* *}$ & $y=1.7703-0.0072 x$ \\
\hline & AP \& yield (Khotkhotia) & $r=-0.98253^{* * *}$ & $y=2.0157-0.0117 x$ \\
\hline & AP \& yield (Ananda) & $r=-0.93221^{* * *}$ & $y=2.1848-0.014 x$ \\
\hline & AP \& yield (Ullash) & $r=-0.92113^{* * *}$ & $y=2.0396-0.0189 x$ \\
\hline & AP \& yield (Tabla) & $\mathrm{r}=-0.91306^{* * *}$ & $y=1.7932-0.0061 x$ \\
\hline
\end{tabular}

${ }^{*} \mathrm{p}<0.05,{ }^{* *} \mathrm{p}<0.01$ and ${ }^{* * *} \mathrm{p}<0.00$.

Oluoch and Chandha (2007) carried out an experiment to determine the yield and quality potential of three cultivated eggplant species (Solanum aethiopium, S. macroempon and S. anguivi) from year 2002 to 2006 in Arusha, Tanzania. 42 selected lines collected from different countries of Africa were evaluated. They got five year mean fruit yield ranging from $1.3 \mathrm{t} /$ ha to $62.5 \mathrm{t} / \mathrm{ha}$ 
with lines AB2, NA, N15, Manyire Green, OAA(089)N18 Heart shape, and N24 giving mean fruits yields of over $57 \mathrm{t} / \mathrm{ha}$. Combind mean yield for species comparison showed that $S$. aethiopium gave significantly higher fruit yields (47.4 $\mathrm{t} / \mathrm{ha}$ ) than other species tested.

The correlations between planting dates and aphid activities have been shown in other crops. Hossain et al. (2002) recorded the early sowing lentil (sown in November) had less aphid (Aphis craccivora) infestation and consequently produced higher yield than the crop sown in later (sown in December). Singh and Dhaliwal (2004) worked on green fodder in Punjab, India and mentioned that highest yield (470.4 quintal/ha) recorded in the crop sown on $10^{\text {th }}$ October when aphid infestation was minimum. They also found minimum green fodder yield (368 quintal/ha) was obtained in plots sown on November 10 showing $21.76 \%$ decrease in fodder yield when aphid infestation was maximum. Gupta and Ram (1989) reported that early sown lucerne yielded maximum, related to least damaging intensity of aphid (Therioaphis maculate Buckton and Aphis craccivora Koch) as compared to later sown. Similarly, Bhaduria et al. (1992) reported that early sown mustard (October 1) was less infested by Lipaphis erysimi (Kalt.) and gave the highest yield. Patel et al. (2004) also recorded that early sowing mustard produced highest yield and this was decreased significantly in delayed sown crop.

\section{CONCLUSION}

The present study shows that early planting eggplants had less aphid infestation and produced better yields than late planting. The eggplant variety Kajla was found to be most suitable among all experimental cultivars (both BARI and local).

Acknoledgements: Thanks are due to the Chairman, Department of Zoology, Rajshahi University, Rajshahi-6205, Bangladesh for providing laboratory facilities.

\section{LITERATURE CITED}

BHADURIA, N.S., BAHADUR, J., DHAM-DHERE, S.V. and JAKHMOLA, S.S. 1992. Effect of different sowing dates of mustard crop on infestation by the mustard aphid Lipaphis erysimi (Kalt.). $J$. Insect Sci. 5: 37-39.

GALLO, O., NAKANA, O., SILVEIRA NETO, S., CARVALHO, R.P.L., BATISTA, G.C., BERTI FILHO, E., PARRA, J.R.P., ZUCCHI, R.A., ALVES, S.B., VANDRAMIN, J.D., MARCHINI, L.C., LOPES, J.R.S. and OMOTOC, C. 2002. Manual de Entomologia Agricola, Piracicaba FEALQ: pp. 1-920.

GU, H., FITT, G.P. and BAKER, G.H. 2007. Invertebrate pests of canola and their management in Australia: a review. Aus.J. Entomol. 46(3): 231-243. 
GUPTA, M.P. and RAM, S. 1989. Effect of dates of sowing and varieties on the incidence of insects pests of Lucerne (Medicago sativa) in India. Tropical Pest Management 35: 399-402.

HOSSAIN, M.A., ALI, M.O., HOSSAIN, M.S., RAHMAN, A.B.M.M. and SHEIKH, M.H.R. 2002. Effect of sowing dates of lentil, Lens culinaris on the infestation of Aphis craccivora Koch and crop yield. Bangladesh J. Entomol. 12(1): 75-84.

KUMAR, G., MEENA, B.L., KAR, R., TIWARI, S.K., GANGOPADHYAY, K.K., BISHT, I.S. and MAHAJAN, R.K. 2008. Morphological diversity in brinjal (Solanum melongena L.) germplasm accessions. Plant Genetic Resources: Characterization and Utilization. 1-5.

OFUYA, T.I. 1997. Control of the cowpea aphid, Aphis craccivora Koch (Homoptera: Aphididae), in cowpea, Vigna unguiculata (L.) Walp. Integrated Pest Management Reviews 2: 199-207.

OLUOCH, M.O. and CHANDHA, M.L. 2007. Evaluation and selection of African eggplant for yield and quality characteristics. Acta Horticult. 752: 303-306

PATEL, S.R., AWASTHI, A.K. and TOMER, R.K.S. 2004. Assessment of yield losses in mustard (Brassica juncea L.) due to mustard aphid Lipaphis erysimi (Kalt.) under different thermal environments in Eastern Central India. Appl. Ecol. and Environ. Res. 2(1):1-5.

REDDY, B.S. and GOWDA, P.M. 1987. Effect of different dates of planting on yield and quality attributes of three brinjal cultivars. South Indian Horticult 35(5): 386-389.

SIDDIKY M.A, HALDER, N.K, ISLAM, Z., BEGUM, R.A. and MASUD, M.M. 2007. Performance of brinjal as influenced by Boron and Molybdenum. Asian J. Plant Sci. 6(2): 389-393.

SINGH, N. and DHALIWAL, J.S. 2004. Effect of date of sowing on oat aphid, Rhopalosiphum padi (Linn.) (Homoptera:Aphididae) and its natural enemies. J. Aphidol. 18: 33-38.

SINGH, N.N. 2006. Mustard aphid and its management. The Aphidological Society of India. pp. 1-52.

SOE, H. 1999. Effect of Pruning on Yield and Quality of Eggplant. ARC Training Report-1999, AVRDC.

WUZHONG, N. 2002. Yield and quality of fruits of solanaceous crops as affected by potassium fertilization. Better Crops International 16(1): 6-8.

(Manuscript received on September 25, 2010; revised on June 6, 2011) 